\title{
A Tale of Two Viruses: Poliomyelitis and COVID-19
}

\author{
Carlos A. Arango, MD
}

n the late 1940s, the American health care system was faced with a crisis. Poliomyelitis outbreaks were crippling American children at record numbers. Public health officials imposed quarantines on cities where polio cases were diagnosed; travel and commerce were also affected. Poliomyelitis struck cities and towns indiscriminately, especially during the summertime. Parents were not letting their children go outside of their homes, use public facilities such as pools, or even go to the beach out of fear of their child becoming ill. Now a novel coronavirus is doing the same to us today.

The poliovirus was the causative agent of this disease, of which there are 3 serotypes. ${ }^{1}$ Humans are the only reservoir for this virus, and it is spread via respiratory secretions and feces. The incubation period for poliomyelitis is 3 to 6 days for onset of infection and 7 to 21 days for developments of paralysis. Approximately $72 \%$ of susceptible children who were infected remained asymptomatic, while another $24 \%$ reported nonspecific symptoms such as low-grade fever, headaches, sore throat, nausea, diarrhea, and malaise. The remaining $1 \%$ to $5 \%$ reported symptoms of viral meningitis (nonparalytic polio) and sometimes developed paresthesia. In less than $1 \%$ of cases of infection, a rapid asymmetric acute flaccid paralysis developed. Most individuals affected by this viral illness recovered quickly without any sequelae, while others experienced partial effects, and a few developed permanent paralysis and even death.

In an attempt to control this illness, health officials instituted sanitation rules: Avoid open drains, avoid unscreened windows, reduce public exposure, and stay away from crowds. Public

\section{AFFILIATION:}

Department of Pediatrics, University of Florida College of Medicine, Jacksonville, Florida

\section{CITATION:}

Arango C. A tale of two viruses: poliomyelitis and COVID-19. Consultant. Published online October 5, 2020. doi:10.25270/con.2020.10.00004

\section{DISCLOSURES:}

The author reports no relevant financial relationships.

\section{CORRESPONDENCE:}

Carlos A. Arango, MD, Department of Pediatrics, Baymeadows Pediatrics, 8399 Bayberry Rd, Jacksonville, FL 32256 (carlos.arango@jax.ufl.edu)

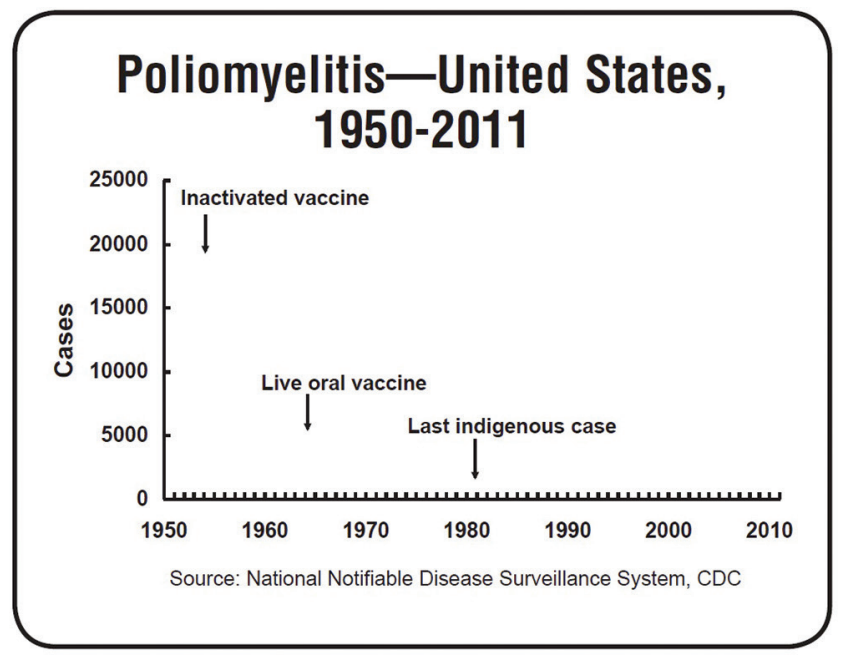

pools were shut down, movie theaters were closed, and schools and summer camps were suspended. These prevention methods were harmless albeit not helpful, since they were the best methods possible given the knowledge available at that time. The modernization of sanitation and drainage work in cities and towns improved the well-being of all inhabitants. The only caveat was that the small exposure to poliovirus in young children provided them an opportunity to mount an immune response to the virus without developing the disease.

Poliomyelitis created feelings of despair and provoked anxiety-it had appeared suddenly around summertime, there was no know reason for its appearance, and no preventive measures were able to stop it. A cure was not yet available for the mysterious illness. Once the disease struck, and the individual developed paralysis, there were few treatment modalities. Multiple therapies were offered, but few of them worked: antispasmodic medication was used to help with muscle contractions, and crutches and walkers were offered. Plasma from individuals who survived the disease was also used. Physical therapy was the mainstay of treatment to help children crippled by this disease. An iron lung was the only modality if the disease progressed to bulbar disease. An average of $2 \%$ to $5 \%$ of children and $15 \%$ to $30 \%$ of adults affected with the disease died. The National Foundation for Infantile Paralysis was created, and its umbrella group, the March of Dimes, was the fundraiser 
organization able to raise money to help people care for a family member who was affected by polio.

In 1949, Harvard physicians John Enders, Thomas Weller, and Frederick Robbins were able to grow poliovirus outside the body using tissue culture of human embryonic muscle and skin cells. For this discovery, these scientists received the 1954 Nobel Prize in Physiology or Medicine. In 1950, Dr Jonas Salk developed a possible vaccine and asked to start the trials for the injectable polio vaccine (IPV) immediately; how-ever, this did not begin until 1954. In parallel, Dr Albert Sabin developed an oral polio vaccine (OPV) in 1956. Trials for this vaccine were started in 1957 . In less than a decade, the number of reported polio cases dropped from more than 20,000 cases per year in the 1950s to only a handful in the 1960s (approximately 161 cases per year)! This discovery was one of the greatest accomplishment of the 20th century (Figure). ${ }^{1}$

Now, move forward to 2020. COVID-19 is affecting the entire world in such a way that had never been though possible. There are numerous similarities between polio and COVID-19. Fear and anxiety is gripping the entire world. Public health measures have been implemented, social distancing has been put into effect, and the use of face masks is ubiquitous. Although a new term is being used- "flattening the curve"measures similar to those of the past with poliomyelitis have been once again urged: shelter in place, avoid pools and movie theaters and going to the beach, and avoid crowds; thus, disruption of commerce is occurring. Economic turmoil is affecting the world's economies. This pandemic must be contained as soon as possible.

This novel coronavirus seems to be spreading by aerosolized respiratory droplets. Avoiding cross-contamination is the current management strategy for the disease. The great majority of affected individuals are asymptomatic, few are developing symptoms, and fewer are becoming severely sick and dying.

Health care officials are using all the resources at their disposal to help contain this novel coronavirus: reducing public exposure, encourage physical distancing from each other, closure of public establishments, mandatory wearing of a face mask, etc. Those measures did not work with poliovirus due to the nature of that virus. However, in multiple countries around the globe, these public recommendations seems to be working in reducing the coronavirus burden.

The use of plasma from recovered individuals has been used both in poliovirus and coronavirus infections, with some anecdotal reports of survival benefit. Multiple medications were or have been trialed in both outbreaks, with varying degrees of success. The development of a polio vaccine has been hailed as one the best discoveries of the century, and it helped pave the way for isolation and manufacturing of vaccines in last several decades. The next logical step is the development of a coronavirus vaccine. Will it work? We do not know; only trials and usage will yield an answer. Today many countries are feverishly working on developing an effective vaccine against COVID-19; some of them are in phase II and phase III. It is hoped that this vaccine(s) will be available to the population worldwide in the next months or years. And it is hoped that this vaccine will have a similar success rate as the polio vaccine did in the past.

The new generation of US parents and physicians have never seen a case of poliomyelitis. The last case was reported in 1979 among an Amish community. Vaccination was the main reason that polio declined dramatically! Just because no cases of polio have occurred in the United States doesn't mean that it cannot return. We need to continue vaccinating our children against all preventable diseases and not let our guard down. This pandemic is affecting our health care system and keeping parents and children away from their health care providers. We need to bring those children back to the doctor's offices and redouble our effort to continue offering and administrating currently recommended vaccinations.

Physicians, nurses, paraprofessionals, emergency medical technicians, respiratory therapists, physical therapists, scientists, and all of my other colleagues in health care: Please do not despair, redouble your efforts, continue researching, allow our scientific community take control of investigations and research, and with time and effort, we shall be rewarded. A new therapy will soon be developed for this virus.

\section{REFERENCE:}

1. Poliomyelitis. In: Hamborsky J, Kroger A, Wolfe C. Epidemiology and Prevention of Vaccine-Preventable Diseases. 13th ed. Centers for Disease Control and Prevention; 2015:297-310. Accessed September 25, 2020. https:// www.cdc.gov/vaccines/pubs/pinkbook/downloads/polio.pdf 